\title{
Deducción y estudio de la ecuación general que determina la cantidad de sustancias no eluidas por cromatografía gaseosa. Análisis de aceites comestibles recalentados.
}

\author{
Por A. Castellón Arnau. \\ Grasas Alimenticias, S. A. - 08520 LLERONA (Barcelona).
}

\section{RESUMEN}

Deducción y estudio de la ecuación general que determina la cantidad de sustancias no eluidas por cromatografia gaseosa. Análisis de aceites comestibles recalentados.

Se deduce' la ecuación general que determina la cantidad de sustancias no eluidas en una separación cromatográfica. Esta ecuación permite el cálculo de dichas sustancias aún cuando el patrón interno no sea de una absoluta pureza y aunque otras sustancias presentes en la muestra sean cromatografiadas junto con él. Es aplicable en la cuantificación por cromatografía de gases de los productos de degradación térmico-oxidativa de los aceites de fritura. Se calcula, asimismo, la desviación estándar teórica de los valores que se obtienen al aplicar dicha ecuación.

El estudio experimental posterior demuestra que se produce una sobrevaloración del porcentaje de sustancias no eluidas si no se tiene en cuenta la presencia de sustancias interferentes.

PALABRAS-CLAVE: Aceite termooxidado - Cromatografía gaseosa - Ecuación de cálculo - Sustancias no eluibles.

\section{SUMMARY}

Deduction and study of the general equation that determines the quantity of non-eluted materials by gas chromatography. Analysis of abused oils.

The general equation that determines the quantity of noneluted materials in a chromatographic separation is deduced. This equation permits the calculation of these materials when the internal standard is not pure and when interfering subtances of the oil are chromatographied with it. The obtained equation is applied in the quantification of the thermal-oxidative degradation products present in heated olis. The theoretical standard deviation of the values of the non-eluted materials is also calculated.

The experimental study shows that there is a positive bias of the non-eluted materials if one does not consider the presence of interfering materials.

KEY-WORDS: Calculating equation - Gas chromatography - Non-eluted materials - Thermooxidixed oil.

\section{INTRODUCCION}

Actualmente está muy difundida la cocción de alimentos por inmersión en baños de aceite comestible. La alta temperatura a que se somete el aceite provoca su oxidación y polimerización térmicas. Las sustancias resultantes de estos procesos disminuyen el valor nutritivo del aceite (1) y pueden resultar tóxicas (2).

Dado que no es posible conocer el grado de deterioro por valoración organoléptica, se impone la determinación química cuantitativa de las sustancias alteradas presentes.

De entre los diversos métodos aplicables destaca la cromatografía de gases cuantitativa por su rapidez y sencillez operativa. Durante la separación cromatográfica de los ésteres metílicos se produce la retención en la columna de la mayoría de las sustancias polares y polímeros formados durante el uso del aceite. Para cuantificar adecuadamente las sustancias que han sido retenidas por la columna, se mezcla con el aceite una determinada cantidad de patrón interno. La proporción de patrón presente en la mezcla antes y después de la cromatografía nos dará la cantidad de sustancias que han sido retenidas en la columna.

Esta técnica es ampliamente utilizada desde hace años (4) (5). No obstante, siempre se ha dado por sentado que las condiciones de análisis son óptimas, es decir, que la pureza del patrón es absoluta y que no existen sustancias interferentes en el aceite que sean cromatografiadas junto con el patrón interno. Dado que, en la mayoría de casos, esto no es así, la aplicación directa de la ecuación simplificada que aparece en las metodologías analíticas dará lugar a resultados incorrectos.

Por ello se deduce aquí la ecuación general aplicable en cualquier caso. 


\section{DEDUCCION MATEMATICA DE LA ECUACION}

Supondremos que todas las sustancias eluibles son cromatografiadas y que las no eluibles son retenidas.

En el caso más general, el patrón interno contiene una cierta proporción de impurezas. Estas impurezas pueden ser no eluibles, quedando retenidas en la columna, y eluibles. Las impurezas eluibles son cromatografiadas con tiempos de retención distintos al del patrón puro y, por tanto, interfieren en el cromatograma de la muestra de aceite.

Asimismo, el aceite puede contener sustancias eluibles que son cromatografiadas al mismo tiempo que el patrón puro y que interfieren con él.

El cálculo del porcentaje de los diferentes ésteres metílicos se realiza por integración y normalización del cromatograma obtenido.

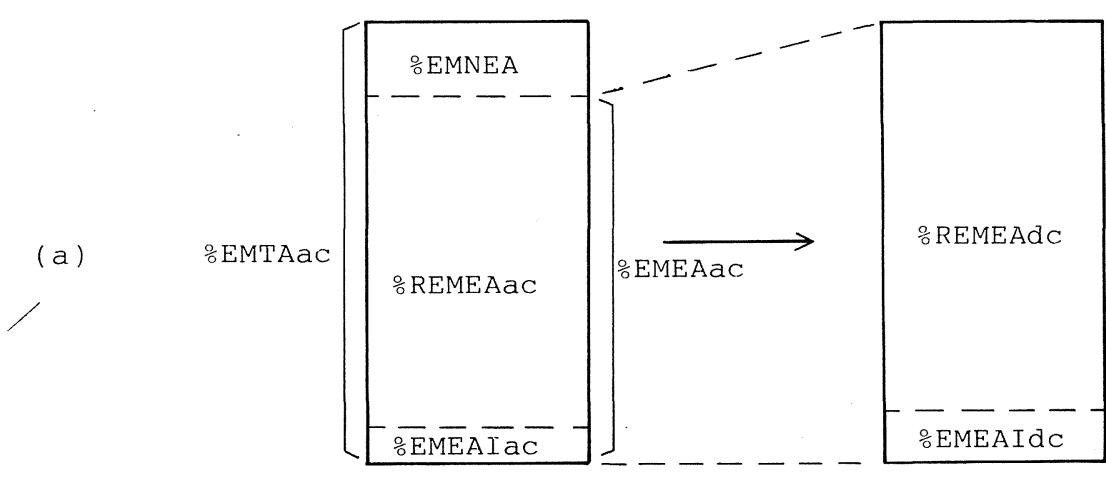

(b)
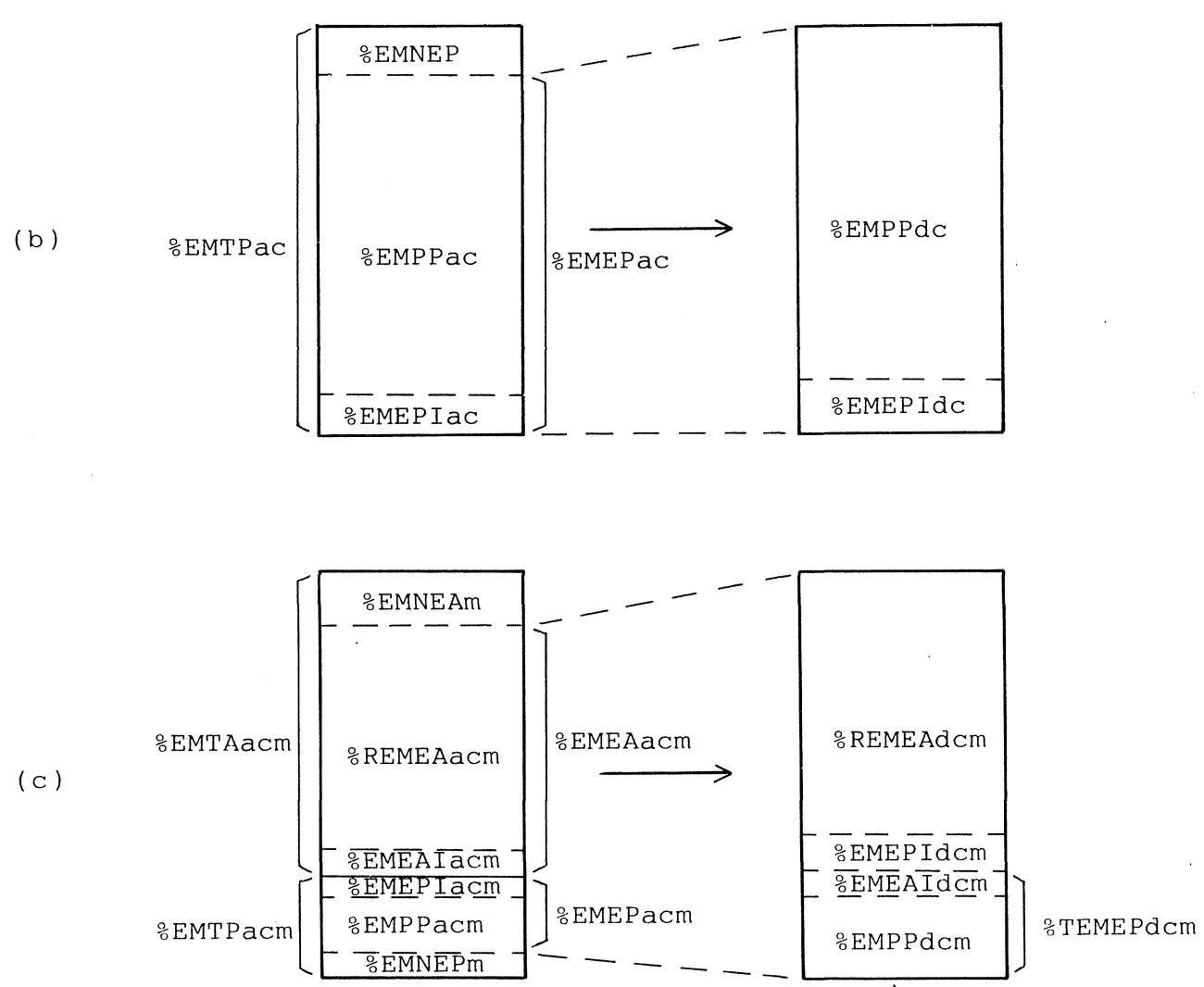

Figura 1

Concentración relativa de las fracciones antes y después de cromatografiar: (a) el aceite metilado, (b) el patrón interno metilado, (c) la mezcla de ambos. 
Para deducir la ecuación general anotaremos, en primer lugar, los conceptos implicados en el cálculo y sus correspondientes simbologías.

EMTA = Esteres metílicos (E.M.) totales del aceite metilado (producto resultante de la metilación completa del aceite).

$E M N E A=E . M$. no eluibles del aceite metilado.

$E M E A=E . M$. eluibles del aceite metilado.

$E M E A I=E . M$. eluibles del aceite metilado interferentes (fracción de los E. M. eluibles del aceite que eluyen junto con el patrón puro).

REMEA $=$ Resto de los E. M. eluibles del aceite metilado (fracción de los E. M. eluibles del aceite que no eluyen con el patrón puro).

$E M T P=E . M$. totales del patrón.

$E M N E P=E . M$. no eluibles del patrón.

$E M E P=E . M$. eluibles del patrón.

$E M E P I=E . M$. eluibles del patrón interferentes (fracción de los E. M. eluibles del patrón que eluyen junto con REMEA).

$E M P P=E . M$. del patrón puro.

En la figura 1 podemos observar la distribución de estas fracciones antes y después de cromatografiar la muestra original metilada, el patrón y la mezcla de ambos. El signo "\%" indica la proporción de esa fracción expresada en tanto por ciento respecto del total. Los sufijos "ac" y "dc" se refieren a las fracciones antes y después de su cromatografía. El sufijo " $\mathrm{m}$ " indica que la fracción se encuentra en la mezcla de aceite y patrón.

Evidentemente, las fracciones EMNEA y EMNEP no se encuentran en las muestras una vez eluidas por to que EMNEAdc y EMNEPdc son nulas. De este modo, simplificaremos la nomenclatura de EMNEAac y EMNEPac llamándolas EMNEA y EMNEP.

La fracción \%TEMEPdcm es el porcentaje correspondiente al total de E.M. eluidos junto con el patrón puro, es decir, este valor será el del patrón puro obtenido tras la cromatografía de la mezcla.

El porcentaje total de E.M. no eluibles (\%TEMNE) será la suma de \%EMNEA y \%EMNEP.

A continuación se indica el desarrollo matemático conducente a la obtención de \%EMNEA.

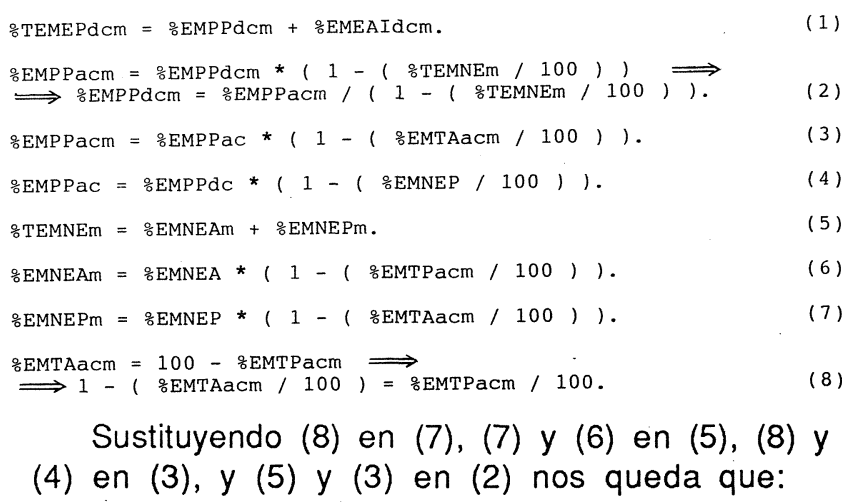

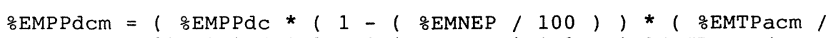
$100)$ ) / ( $1-($ ( \& EMNEA * ( $1-($ \&EMTPacm ) $100)+($ \&EMNEP *( $)$ EMTPacm $/ 100)) /(100))$.

Del mismo modo, para \%EMEAldcm:

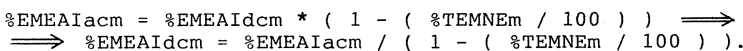

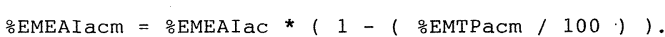

¿EMEAIac $=\frac{\circ}{6}$ EMEAIdc $*\left(1-\left(\frac{\circ}{0}\right.\right.$ EMNEA $\left.\left./ 100\right)\right)$.

Sustituyendo (12) en (11), y (11) y la ecuación desarrollada de (5) en (10) nos queda que:

EMEAIdcm $=($ OEMEAIdc * $(1-($ OEMNEA $/ 100)$ ) $*(1-$ ( 5 EMTPacm / 100$)$ ) +( $(00), 1,100)$ ).

Por otro lado:

$($ \&EMTPacm $/ 100)=($ pEMP $/($ pEMP + pEMA $))$

Siendo: $\mathrm{pEMP}=$ Peso de patrón interno metilado en la mezcla.

pEMA = Peso de aceite metilado en la mezcla.

Por fin, sustituyendo (13) y (9) en (1), desarrollando, sustituyendo (14) en la ecuación obtenida y reordenando, nos queda que:

$\therefore$ EMNEA $=\left(1+\right.$ pEMP $/$ pEMA * $\left(1+\left(\frac{2}{5}\right.\right.$ EMEAIdc $-\frac{2}{5}$ EMPPdc -

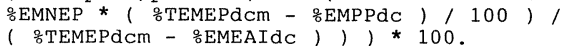

La ecuación (15) nos da el valor de la cantidad de ésteres metílicos no eluidos de la columna en el caso de que el patrón metilado no sea de $100 \%$ de pureza y de que el aceite metilado posea sustancias que interfieran con el patrón puro. Además, esta ecuación tiene en cuenta la cantidad de sustancias no eluibles que puede llevar el patrón. No obstante, esta cantidad debe ser medida utilizando otro patrón que no las lleve, como referencia. Así, no resulta conveniente utilizar patrones con sustancias no eluibles puesto que hace necesario su análisis previo, introduciendo un importante error de medida en el resultado final. 
En el caso de que el patrón utilizado no lleve sustancias no eluibles, \%EMNEP es nulo y la ecuación (15) queda:

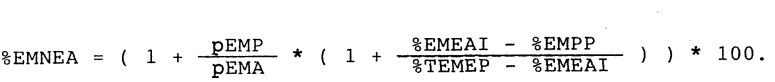

Observemos que han sido eliminados los sufijos para simplificar.

Si el patrón es de una absoluta pureza y los E.M. de la muestra a analizar no contienen proporción alguna de sustancias que puedan interferir con el patrón puro, \%EMPP será 100 y \%EMEAI nulo con lo que la ecuación anterior queda finalmente:

\%EMNEA $=\left(1+\frac{\text { pEMP }}{\text { pEMA }} *\left(1-\frac{100}{8 \text { TEMEP }}\right)\right) * 100$.

Esta ecuación es idéntica a la dada por otros autores (4) (5). No se alude en estas referencias, sin embargo, a su carácter simplificado ni se indica la variación que puede sufrir el \%EMNEA en el caso de que no se den las circunstancias anteriores. Es de notar, de la observación de la ecuación (16), que si bien la pureza del patrón influirá poco en el resultado final, un valor de \%EMEAI del orden de la unidad, inducirá un resultado muy diferente del real.

Para llevar a cabo el cálculo precedente, hemos supuesto que se partía de un patrón metilado y de una muestra igualmente metilada. Dado que la muestra objeto de estudio se encuentra normalmente sin metilar, deberíamos derivatizarla y purificarla antes de realizar el análisis. Este proceso de síntesis y posterior mezcla con el patrón metilado puede provocar, no obstante, un error en el resultado final debido a la posible diferencia en la proporción de sustancias no eluibles antes y después de realizar la metilación. De este modo, si no podemos asegurar que esto no ocurrirá, es preferible partir de una mezcla de aceite y patrón sin metilar y derivatizarlos juntos. En este caso, hemos de tener en cuenta que la humedad, las impurezas y el insaponificable, serán contabilizados entre las sustancias no eluibles dado que, o son eliminados durante el proceso de metilación, o son retenidos por la columna, o no son cuantificados por el detector.

Partiendo de los pesos de aceite y de patrón sin metilar tendremos:

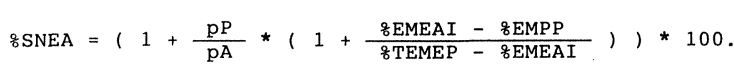

\section{Siendo:}

$\%$ SNEA = Porcentaje de sustancias no eluibles del aceite.

$\mathrm{pP}=$ Peso de patrón sin metilar.

$\mathrm{pA}=$ Peso de aceite sin metilar.

Al comparar el \%SNEA con el \%EMNEA observamos que en el cálculo del \%SNEA se parte de materia grasa bruta y no de sus E. M. Esto hace que aparezca una diferencia entre ambos resultados debida al distinto peso de los E. M. respecto del peso de aceite original. Esta diferencia es calculable ya que, aceptando que el aceite está formado mayoritariamente por triglicéridos, la relación entre el peso de una muestra y el de sus E.M. será:

$\frac{\mathrm{pA}}{\mathrm{pEMA}}=\frac{92+3 *(\text { PMaga }-18)}{3 * \text { PMema }}$

Siendo:

PMaga $=$ Peso molecular medio de los ácidos grasos del aceite.

PMema $=$ Peso molecular medio de los E. M. del aceite.

Por otro lado: $\quad$ PMàga $=$ PMema -14

Sustituyendo (20) en (19) y despejando, nos queda que:

$\mathrm{pA}=\frac{\text { PMema }-4 / 3}{\text { PMema }} *$ pEMA

De igual modo para el patrón obtenemos:

$\mathrm{pP}=\frac{\mathrm{PMemp}-4 / 3}{\mathrm{PM} \text { emp }} * \mathrm{pEMP}$

A partir de estas ecuaciones se deduce que: (23)

(24)

$\frac{\mathrm{pP}}{\mathrm{pA}}=\mathrm{f} * \frac{\mathrm{pEMP}}{\mathrm{pEMA}} ;$ siendo $\mathrm{f}=\frac{\mathrm{PMema} *(\text { PMemp }-4 / 3)}{\mathrm{PMemp} *(\text { PMema }-4 / 3)}$

La diferencia entre los valores de \%EMNA y \%SNEA se obtendrá sustituyendo (23) en (18) y restando el resultado a (16):

\&EMNEA- 8 SNEA $=(1-f) * \frac{\text { pEMP }}{\text { pEMA }}\left(1+\frac{\frac{8}{8} \text { EMEAI }-\frac{8}{8} \text { EMPP }}{8 \text { TEMEP }-\frac{8}{8} \text { EMEAI }}\right) * 100$.

Puesto que el \%EMNEA sólo puede ser positivo o nulo vemos que, a partir de la ecuación (16):

(26)

$-100<\frac{\text { pEMP }}{\text { pEMA }} *\left(1+\frac{8 \text { EMEAI }-8 \text { EMPP }}{\text { कTEMEP }-8 \text { EMEAI }}\right) * 100<0$

Con lo que:

$\mid$ \&EMNEA - o SNEA $|<|(\mathrm{f}-1) * 100 \mid$

Los aceites vegetales y animales normalmente utilizados poseen un PMema comprendido entre 221 -aceite de coco- y 324 -aceite de colza alto en erúcico- y si utilizamos la triheptadecanoína, 
con un PMemp de 284, como patrón interno obtenemos un valor de $f$ comprendido entre 1.00135 y 0.99942 con lo que las diferencias entre \%SNEA y \%EMNEA, debidas a la variación del peso molecular del aceite después de metilar, serán inferiores a 0.13 .

En definitiva, si la metilación y la extracción son completas o afectan en la misma proporción a las sustancias eluibles del aceite y del patrón, podremos calcular el \%SNEA a sabiendas de que la diferencia con el \%EMNEA debida al proceso de metilación será despreciable pero teniendo en cuenta que incluirá al porcentaje de humedad, impurezas e insaponificables.

\section{DESVIACION ESTANDAR DEL \%EMNEA}

La ecuación (16) no nos da idea, por sí misma, de su precisión. Es necesario calcular la desviación estándar de su resultado para conocer cual es la dispersión teórica y dentro de qué intérvalo se hallará el valor real.

Si suponemos que los aparatos utilizados balanza analítica y cromatógrafo de gases-dan respuestas cuyas desviaciones se ajustan a la distribución normal y que todas las variables de la ecuación (16) son independientes, el primer desarrollo de la fórmula de Taylor nos da una aproximación del valor de la varianza (6):

$$
\begin{aligned}
& \mathrm{s}_{\text {\%EMNEA }}^{2}=\left(\frac{\partial \text { \&EMNEA }}{\partial \text { pEMA }}\right)^{2} * \mathrm{~s}_{\mathrm{pEMA}}^{2}+\left(\frac{\partial \text { \&EMNEA }}{\partial \text { pEMP }}\right)^{2} * \mathrm{~s}_{\mathrm{pEMP}}^{2}+
\end{aligned}
$$

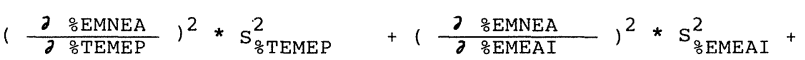

$$
\begin{aligned}
& \left(\frac{\partial \% \text { EMNEA }}{\partial \text { EMPP }}\right)^{2} * \mathrm{~s}_{\frac{\mathrm{g}}{8} \mathrm{EMPP}}^{2}
\end{aligned}
$$

en nuestro caso:

$$
\begin{aligned}
& \frac{\partial \text { OEMNEA }}{\partial \text { PEMA }}=-\frac{\frac{\circ}{8} \text { EMNEA }-100}{\text { pEMA }} \\
& \frac{\partial \text { \%EMNEA }}{\partial \text { pEMP }}=\frac{\text { \%EMNEA }-100}{\text { pEMP }} \\
& \frac{\partial \text { IEMNEA }}{\partial \text { \&TEMEP }}=-\frac{\text { PEMP }}{\text { pEMA }} * \frac{\text { क्EMEAI }- \text { \&EMPP }}{(\text { gTEMEP }- \text { \&EMEAI })^{2}} * 100 \\
& \frac{\partial \text { \&EMNEA }}{\partial \text { \&EMEAI }}=\frac{\text { \&EMNEA }-100}{\left(\frac{8}{8 T E M E P}-\text { \&EMEAI }\right)} \\
& \frac{\partial \text { \&EMNEA }}{\partial \text { \&EMPP }}=-\frac{\text { pEMP }}{\text { pEMA }} * \frac{100}{(\text { \&TEMEP }- \text { कEMEAI })}
\end{aligned}
$$

Sustituyendo en la ecuación (28) y despejando nos queda que la desviación estandar del \%EMNEA es:

\footnotetext{
$S_{\text {gEMNEA }}=\left(\left(\frac{\text { \&EMNEA-100 }}{\mathrm{pEMP}} * \mathrm{~S}_{\mathrm{pEMP}}\right)^{2}+\left(\frac{\text { \&EMNEA-100 }}{\mathrm{pEMA}} * \mathrm{~S}_{\mathrm{pEMA}}\right)^{2}+\right.$

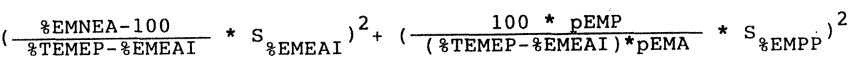
$\left.+\left(\frac{\left(\frac{8}{8} \text { EMEAI }-8 \text { EMPP }\right) * \text { pEMP }}{\left(\frac{8}{8} \text { TEMEP }-\frac{8}{8} \text { EMEAI }\right) * \text { pEMA }} * \mathrm{~S}_{8 \text { TEMEP }}\right)^{2}\right)^{1 / 2}$.

Los términos $S_{\text {PEMA }}$ y $S_{\text {pEMP }}$ corresponden a las desviaciones estándar de las pesadas de los E. M. del aceite y del patrón respectivamente.

$S_{\% \text { EMEAI }}, S_{\% \text { TEMEP }}$ y $S_{\% \text { EMPP }}$ son las desviaciones estándar de los valores hallados para el \%EMEAI, \%TEMEP y \%EMPP, y dependerán de las características y condiciones del cromatógrafo.

De igual modo se deduce la ecuación de la desviación estándar del \%SNEA sin más que sustituir los pEMA y pEMP por los $\mathrm{pA}$ y $\mathrm{pP}$ obteniéndose:

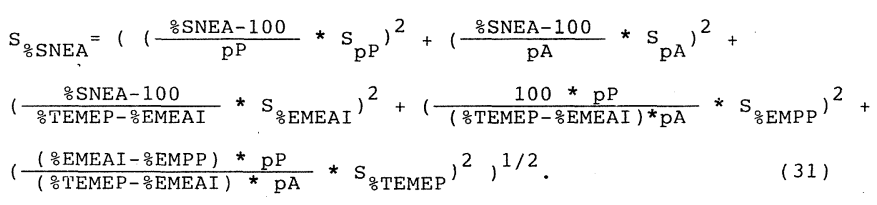

\section{PARTE EXPERIMENTAL}

Vamos a estudiar, ahora, la validez de las ecuaciones generales deducidas (16) y (18) y el ajuste obtenido mediante las ecuaciones (30) (31). Con este fin, analizaremos una muestra de aceite refinado de manteca de cerdo, obtenida friendo 100 gramos de patatas lavadas y cortadas sin pelar en una freidora doméstica que contenía inicialmente $4.5 \mathrm{Kg}$ de aceite. Las patatas se sumergieron en el aceite a $190^{\circ} \mathrm{C}$ durante 4 minutos a intérvalos de 30 minutos en periodos de 8 horas durante dos días. Tras la segunda jornada, se extrae una muestra de 50 gramos de aceite que se conserva en atmósfera de nitrógeno a $-20^{\circ} \mathrm{C}$. Las determi"naciones efectuadas antes y después del tratamiento térmico -ver tabla I- son:

- Acidez (7)

- Peróxidos (8)

- Color GARDNER (9)

- Humedad (10)

- Impurezas sólidas (11)

- Insaponificables (12)

- Perfil de ácidos grasos (13)

- Polímeros y productos de oxidación de aceites vegetales recalentados, por cromatografía de gases (5)

- Polímeros y productos de oxidación de aceites recalentados, por cromatografía de gases (\%EMNEA y \%SNEA)

Los ésteres metílicos se analizaron en un cromatógrafo de gases marca Perkin-Elmer modelo 8310 provisto de un detector de ionización de 
Tabla I

Características del aceite de manteca antes y después del proceso de fritura.

\begin{tabular}{lcc}
\hline & M.0 & M.16 \\
\hline Grado de acidez (\% oleico) & 0.23 & 0.38 \\
Indice de Peróxidos (meq O/Kg) & 1.25 & 4.25 \\
Color GARDNER & $2-3$ & $6-7$ \\
Humedad (\%) & 0.02 & Tr. \\
Impurezas sólidas (\%) & Tr. & Tr. \\
Insaponificables (\%) & 0.10 & 0.13 \\
Acidos grasos (\%): & & \\
C 14.0 & 1.6013 & 1.8899 \\
C 16.0 & 20.0643 & 20.6049 \\
C 16.1 & 4.6430 & 4.9130 \\
C 17.0 & 0.4010 & 0.4564 \\
C 17.1 & 0.5460 & 0.5402 \\
C 18.0 & 4.9608 & 5.0440 \\
C 18.1 & 52.3677 & 52.7362 \\
C 18.2 & 11.7723 & 10.6313 \\
C 18.3 & 0.9071 & 0.6704 \\
C 20.1 & 1.1289 & 1.1016 \\
C 20.2 & 0.5336 & 0.5317 \\
Otros & 1.0740 & 0.8804 \\
\hline
\end{tabular}

Abreviaturas:

M.0: Aceite de Manteca.

M.16: Id. tras $16 \mathrm{~h}$ de fritura.

llama y en las siguientes condiciones cromatográficas:

- Columna: DEGS al $10 \%$ en Supelcoport $80 / 100$ de $2 \mathrm{~m}$ de longitud y $1 / 8$ " de $\mathrm{d}$. i.

- Temperatura: $185^{\circ} \mathrm{C}$ durante 10 minutos, aumentando a $215^{\circ} \mathrm{C}$ en $1 \mathrm{~min}$. y manteniéndola $7.5 \mathrm{~min}$.

- Flujo de $\mathrm{N}: 30 \mathrm{ml} / \mathrm{min}$.

Para el cálculo cuantitativo de los ésteres metílicos se aplicaron los factores de respuesta teóricos de Ackman y Sipos (14).

Antes de proceder al análisis del \%EMNEA se determinan las purezas de los patrones internos, la cantidad inicial de \%EMEAldc y las desviaciones estándar de cada medida.

La desviación estándar correspondiente a una pesada $\left(S_{p}\right)$ en la balanza analítica utilizada en este trabajo es de $0.0001 \mathrm{gr}$. Para preparar las mezclas de aceite y patrón se procedió en el siguiente orden de pesadas:

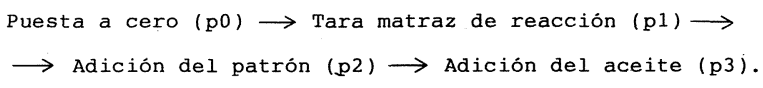

Como las pesadas se realizan consecutivamente sobre el mismo matraz:

$$
S_{p 1}=S_{p 2}=S_{p 3}=\left(S_{p}^{2}+S_{p}^{2}\right)^{1 / 2}=2^{1 / 2} * 10^{-4}
$$

Las desviaciones estándar de los pesos del aceite y del patrón y de sus $E$. M. serán:

$$
\begin{aligned}
& S_{p E M P}=S_{p P}=\left(S_{p 1}^{2}+s_{p 2}^{2}\right)^{1 / 2}=2 * 10^{-4} \\
& S_{p E M A}=S_{p A}=\left(S_{p 2}^{2}+s_{p 3}^{2}\right)^{1 / 2}=2 * 10^{-4}
\end{aligned}
$$

Como patrón interno se ha utilizado triheptadecanoína SIGMA (T-2151) para el análisis del aceite y heptadecanoato de metilo MERCK (9754) para el análisis de los E. M. del aceite. El cálculo de la desviación estándar del \%EMPP se ha realizado a partir de 6 cromatogramas consecutivos de cada patrón interno obteniéndose:

$\mathrm{S}_{\% \text { EMPP }}=0.0564$

La pureza del patrón interno hallada es $99.88 \%$ para la triheptadecanoína (TH) y 99.05 para el heptadecanoato de metilo (HM). Dado que la desviación calculada se refiere a una sola medida y como que la pureza hallada es la media de seis medidas, la desviación estándar de la pureza del patrón interno será:

$$
S_{\% \text { EMPP }}=0.0564 / 6^{1 / 2}=0.0230
$$

El cálculo de las desviaciones estándar del \%TEMEP y del \%EMEAl es más complejo. Para realizarlo se representó, inicialmente, la distribución de las diferencias de los duplicados de 400 análisis cromatográficos obteniéndose la dispersión de puntos de la figura 2. Dado que esta distribución de puntos no es muy clara, se procedió a la representación de las diferencias relativas -figura 3-. En este caso sí se puede observar claramente que a valores de concentración comprendidos entre 4 y $52 \%$, el $95 \%$ de los puntos se halla bajo una curva hiperbólica de ecuación $y=0.640 / x$. El hecho de que la curva sea hiperbólica nos indica que la diferencia entre duplicados es inferior, en el $95 \%$ de los casos, a un valor constante igual a 0.640 -ver figura 2-. Para valores inferiores al $4 \%$ se observa, no obstante, que la distribución de los puntos es distinta. De la figura 2 podemos observar que la práctica totalidad de los puntos a concentraciones inferiores al $4 \%$ se encuentra bajo la curva representada.

Si aceptamos que la distribución de las diferencias sigue una distribución normal para cada concentración y dado que el $95 \%$ de estas dife- 


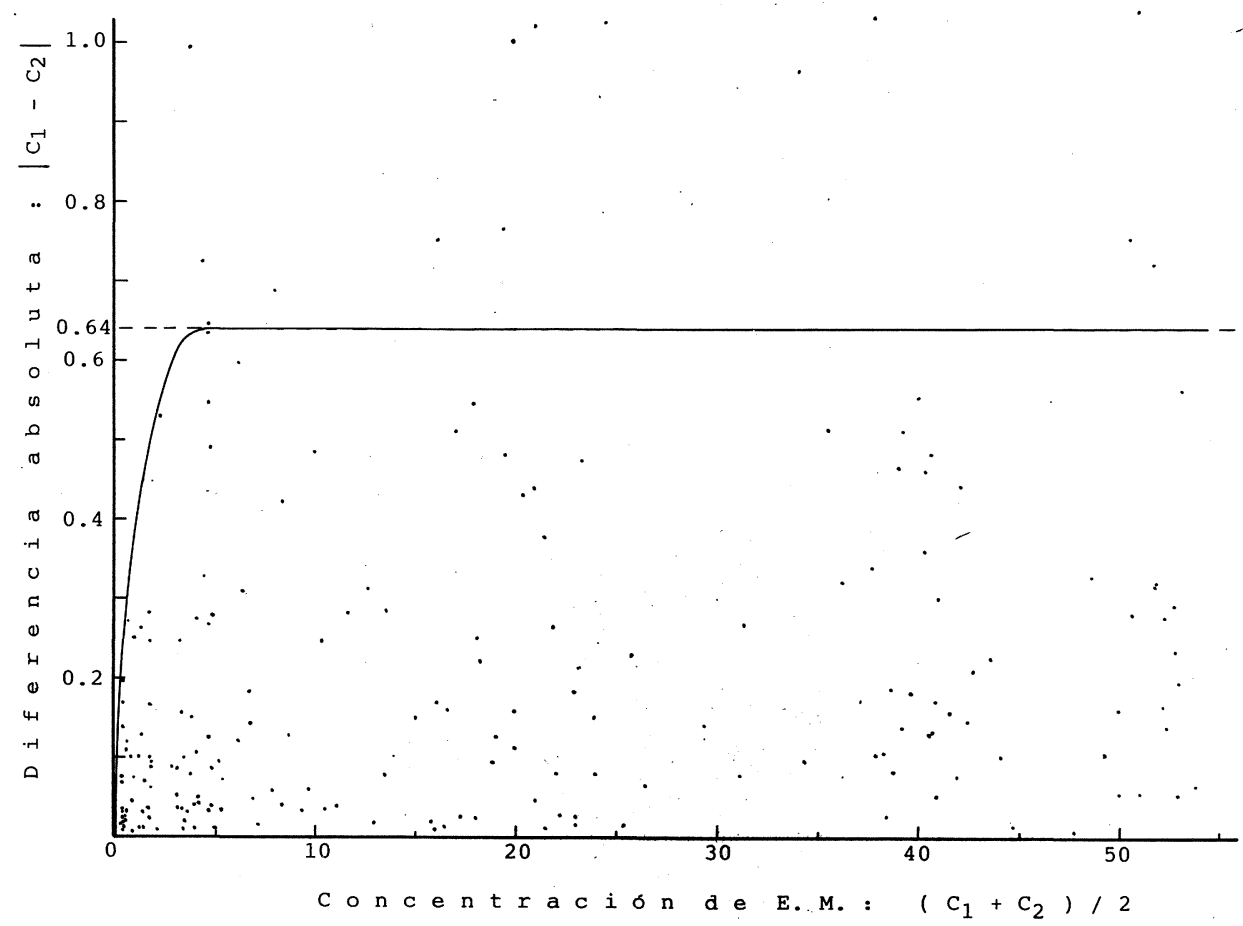

Figura 2

Distribución de las diferencias absolutas entre duplicados de la misma concentración de E. M. C, = Porcentaje del primer duplicado; $\mathrm{C}_{2}=$ Porcentaje del segundo.

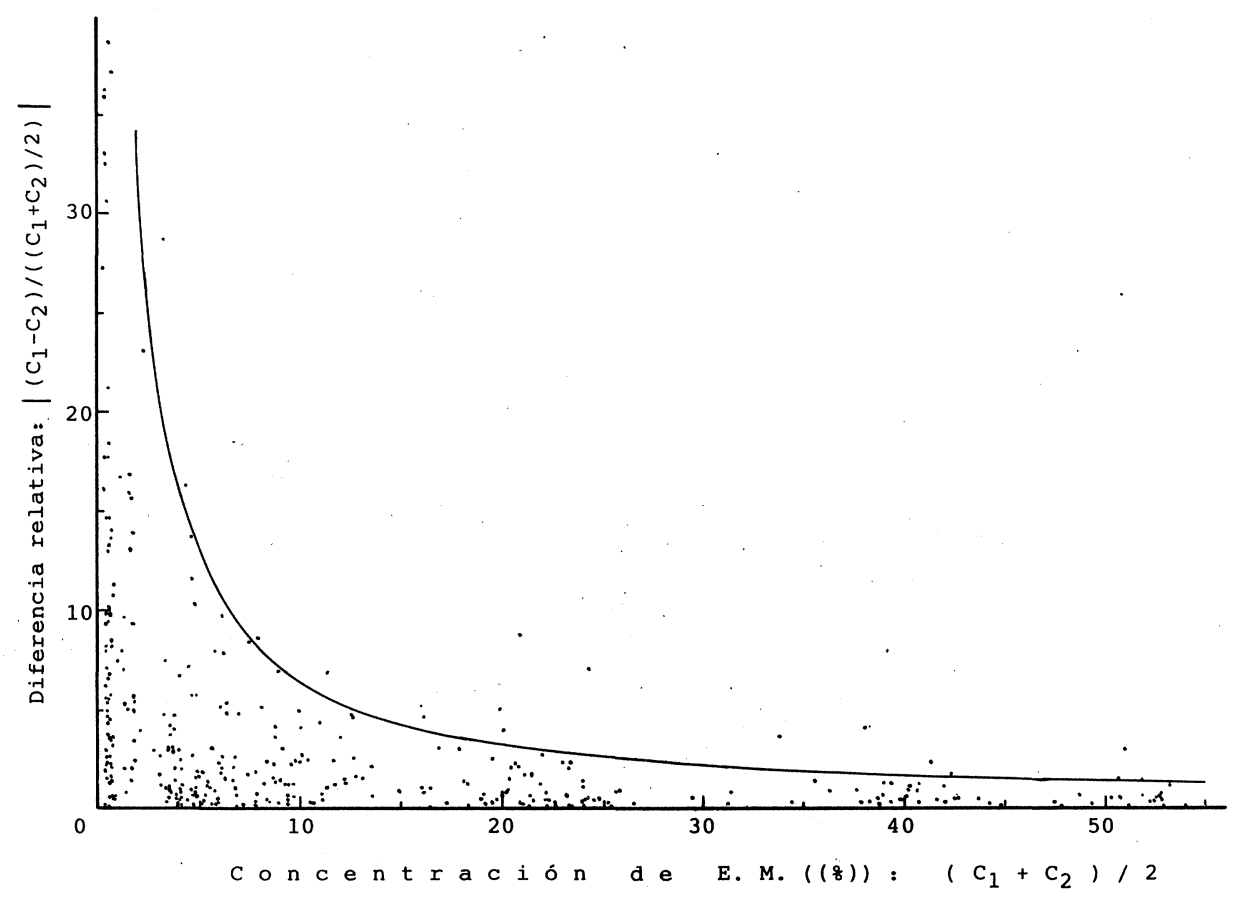

Figura 3

Distribución de las diferencias relativas entre duplicados de la misma concentración de E. M. 
rencias es inferior a 0.640 , la desviación estándar de las diferencias entre duplicados será:

$$
\mathrm{Sd}=0.640 / 1.960=0.3265
$$

y la desviación estándar de las medidas individuales será:

$$
S i^{2}=S d^{2} / 2 \Rightarrow S i=S_{\% \text { TEMEP }}=0.2309
$$

para valores de \%TEMEP comprendidos entre el 4 y el $52 \%$.

La desviación estándar del \%EMEAl se ha determinado a partir de la gráfica 2 observando que la diferencia entre duplicados para una concentración del $1 \%$ es inferior a 0.400 por lo que, como antes:

$$
\begin{aligned}
& \mathrm{Sd}=0.400 / 1.960=0.2041 \Rightarrow \\
& \mathrm{Si}=\mathrm{S}_{\text {\%EMEAI }}=0.1443
\end{aligned}
$$

La cantidad de \%EMEAl hallada en el aceite es de 0.9966 y ha sido calculada a partir de 4 determinaciones. Como en el caso del patrón interno, la desviación estándar de este valor será:

$$
S_{\% \text { EMEAI }}=0.1443 / 4^{1 / 2}=0.0722
$$

En la figura 4 se indica la variación de la desviación estándar del \%EMNEA a diferentes concentraciones de patrón interno. En ella se puede ver que la concentración que da lugar a la desviación más baja -concentración óptima- se sitúa en torno al $50 \%$ y que al preparar $100 \mathrm{mg}$ de mezcla en lugar de 50 se reduce considerablemente el error experimental.

Con el fin de reducir el consumo de reactivo, podemos partir de una mezcla al $40 \%$ de patrón interno sin que se aumente sensiblemente la desviación estándar del \%EMNEA.
A fin de conocer la validez de las ecuaciones (16), (18), (30) y (31), ensayar la concentración óptima de patrón y estudiar la conveniencia de partir del aceite a estudiar tal cual o de hacerlo previa metilación, se procedió a analizar el \%SNEA en las condiciones indicadas en el correspondiente método de la AOAC (5) partiendo de $10 \mathrm{mg}$ de $\mathrm{TH}$ y $40 \mathrm{mg}$ de aceite, y de $40 \mathrm{mg}$ de TH y $60 \mathrm{mg}$ de aceite. Igualmente, se realizó el análisis del $\%$ EMNEA partiendo de los E. M. de la muestra obtenidos según las recomendaciones de la AOAC (13) y mezclándolos con $\mathrm{HM}$ en las mismas proporciones que en el ensayo anterior.

\section{RESULTADOS Y DISCUSION}

Se prepararon mezclas por duplicado del aceite y la triheptadecanoína al 20 y $40 \%$, realizándose cuatro análisis cromatográficos en cada mezcla. Del mismo modo se procedió con las mezclas de los E. M. del aceite y el heptadecanoato de metilo.

Los valores medios de los resultados obtenidos así como sus desviaciones estándar experimentales y teóricas se detallan en la tabla II. A partir de estos valores se observan algunos hechos interesantes:

- Existe una diferencia apreciable entre los porcentajes de sustancias no eluidas obtenidos a partir de la ecuación de la AOAC (\%NEM) y de la desarrollada aquí (\%SNEA).

- El valor de \%EMNEA obtenido partiendo de los $E$. M. es significativamente $(p<0.01)$ muy inferior al \%SNEA obtenido partiendo

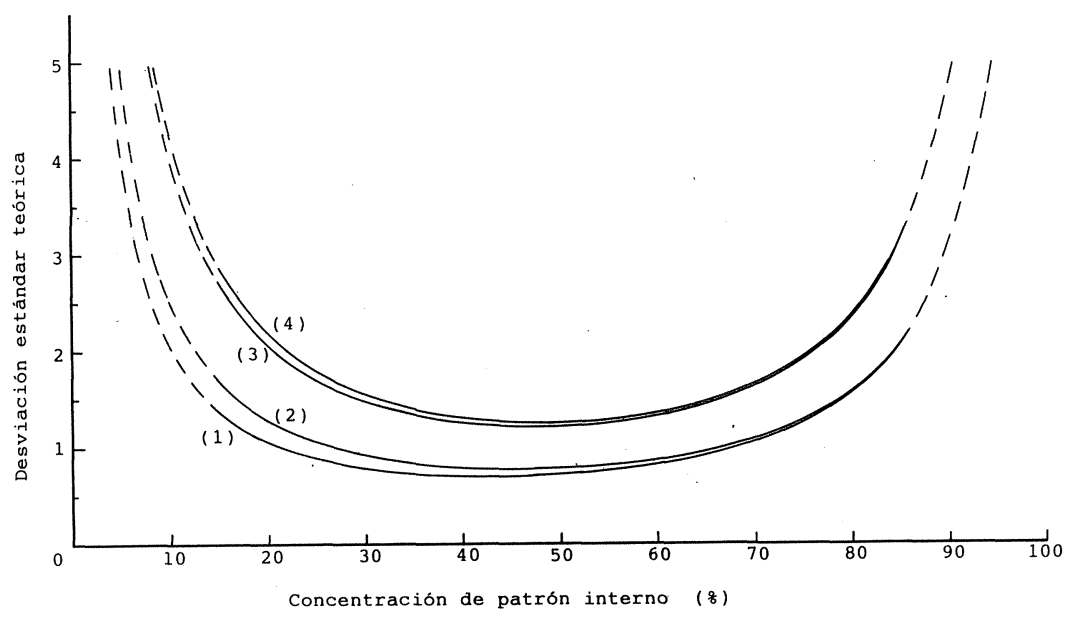

Figura 4

Variaciones de la desviación estándar teórica del \%EMNEA a diferentes concentraciones de patrón interno en caso de: (1) peso total $(\mathrm{PT})=100 \mathrm{mg}$, sustancias interferentes $(\mathrm{SI})=0 \% ;(2) \mathrm{PT}=100 \mathrm{mg}, \mathrm{SI}=1 \% ;(3) \mathrm{PT}=50 \mathrm{mg}$, $S I=0 \%$; (4) PT $=50 \mathrm{mg}, S I=1 \%$. 
Tabla II

Valores experimentales de las sustancias no eluidas obtenidos aplicando la ecuación que aparece en el método de la AOAC y la ecuación general deducida aqui y sus desviaciones estándar experimentales y teóricas.

\begin{tabular}{|c|c|c|c|c|}
\hline & $\mathrm{ATH} 20$ & АTH 40 & EMHM 20 & EMHM 40 \\
\hline$\overline{\circ S N E A}$ & 9.64 & 8.17 & - & - \\
\hline \%EMNEA & - & - & 5.32 & 4.98 \\
\hline$\overline{\frac{O}{0} \mathrm{NEM}}$ & 12.69 & 9.21 & 7.53 & 4.96 \\
\hline$\ldots \ldots$ & $\ldots \ldots$ & $\ldots \ldots$ & $\ldots \ldots$ & $\ldots \ldots$ \\
\hline 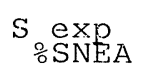 & 1.21 & 0.67 & - & - \\
\hline $\begin{array}{l}\text { S exp } \\
\text { 。MNEA }\end{array}$ & - & - & 0.60 & 0.61 \\
\hline $\begin{array}{l}\text { S teor } \\
\text { 。 SNEA }\end{array}$ & 1.91 & 0.68 & - & - \\
\hline $\begin{array}{l}\text { S teor } \\
\text { \%EMNEA }\end{array}$ & - & - & 2.00 & 0.70 \\
\hline
\end{tabular}

Abreviaturas:

ATH 20: Mezcla de aceite y triheptadecanoina al $20 \%$

$\mathrm{ATH} 40$ : Id. al $40 \%$

EMHM 20: Mezcla de los E.M. del aceite y el heptadecanoato de metilo al $20 \%$

EMHM 40: Id. al $40 \%$.

OSNEA: Valor medio de los porcentajes de sustancias no eluidas obtenidos aplicando la ecuación deducida aquí.

ㄷMNEA: Valor medio de los porcentajes de ésteres metilicos no eluidos obtenidos aplicando la ecuación deducida aquí.

NN: Valor medio de los porcentajes de sustancias no eluidas obtenidos al aplicar la ecuación de la AOAC.

S exp: Desviación estándar calculada a partir de los porcentajes -SNEA de sustancias no eluidas hallados experimentalmente.

S exp: Desviación estándar calculada a partir de los porcentajes

oEMNEA de E.M. no eluidos hallados experimentalmente.

S teor: Desviación estándar teórica prevista según la

oSNEA ecuación (30).

S têror Desviación estándar teórica prevista según la ecuación (31).

de la muestra original aún sumando los porcentajes de humedad, impurezas e insaponificables.

- Las desviaciones estándar obtenidas en las mezclas de $60 \mathrm{mg}$ de muestra y de $40 \mathrm{mg}$ de patrón interno (ATH 40 y EMHM 40) son del. orden de las teóricas. Las obtenidas en las mezclas al $20 \%$ (ATH 20 y EMHM 20) son inferiores a las esperadas teóricamente.

De estas observaciones se deduce que:

- La ecuación utilizada en el cálculo de las sustancias no eluibles que aparece en el método n.9 977.17 de los "Official Methods of Analysis (1990)" de la AOAC proporciona resultados muy altos si no se tienen en cuenta las sustancias interferentes del aceite a analizar que aparecen en la zona de elución del patrón interno. Estas desviaciones alcanzan valores de hasta 4 unidades cuando la concentración de estas sustancias se sitúa en torno al $1 \%$.

- Durante el proceso de preparación y purificación de los E. M. se pierde una importante cantidad de sustancias no eluibles. 
- Partir de 100 mg de mezcla en lugar de 50 utilizando una proporción del $40 \%$ de patrón interno, no parece mejorar, en general, la dispersión de los resultados a pesar de la predicción teórica.

\section{CONSIDERACIONES FINALES}

La ecuación general para el cálculo del porcentaje de sustancias no eluidas deducida aquí, es aplicable a cualquier tipo de aceite tanto de origen vegetal como animal.

La ecuación de la desviación estándar teórica da resultados en concordancia con los valores obtenidos experimentalmente.

La modificación propuesta $-40 \%$ patrón interno y $100 \mathrm{mg}$ peso total- no parece mejorar significativamente la precisión del método original a pesar de la predicción teórica. Esta última consideración debería, no obstante, ser objeto de un estudio experimental más amplio.

\section{AGRADECIMIENTOS}

A los profesores Olga Juliá y Frederic Otset del Departamento de Estadística Matemática de la Universidad Central de Barcelona por su colaboración en el cálculo de la ecuación de la desviación estándar teórica.

\section{BIBLIOGRAFIA}

1. Jimenez, S.; Rodriguez Suarez, A.; Vidaud Candebat, Z.; Grata González, E.; Morán, J. L. y Torres, E.- "Digestibilidad in vivo de la manteca de cerdo sobrecalentada".Grasas y Aceites 37 (1986) 194-196.

2. Jiménez, S.: Vidaud, Z.; Rodríguez, A.; Gómez, R. y Piñón, D.- "Toxicidad subaguda de las mantecas de freidura. I. Caracterización química y evaluación de algunos indicadores clínicos y hematológicos en ratas".- Grasas y Aceites 38 (1987) 363-366.

3. Ruiz Jiménez, V.- "Aspectos toxicológicos y nutricionales de los ácidos grasos alterados por los tratamientos industriales de los aceites comestibles".- Grasas y Aceites 38 (1987) 326-335.

4. Waltking, A. E.; Seery, W. E. and Bleffert, G. W.- "Chemical Analysis of Polimerization Products in Abused Fats and Oils".- J. Am. Oil Chemists' Soc. 52 (1975) 96-100.

5. Official Methods of Analysis of the AOAC.- 15th Edition (1990).- Method 977.17.

6. Apostol, T. M.- "Mathematical Analysis".- Ed. AddisonWesley. Massachusets.

7. Norma UNE 55-011.- "Determinación de la acidez libre".Instituto Español de Normalización (IRANOR).

8. Norma UNE 55-023.- "Determinación del Indice de Peróxidos". - Instituto Español de Normalización (IRANOR).

9. 1933 GARDNER Scale (A.S.T.M. Specification D154 (1933)).

10. Norma UNE 55-020.- "Determinación de Humedad y Materias Volátiles". - Instituto Español de Normalización (IRANOR).

11. Norma UNE 55-002.- "Determinación de Impurezas con Eter de Petróleo". - Instituto Español de Normalización (IRANOR).

12. Norma UNE 30-305.- "Determinación del Insaponificable Método del Eter de Petróleo".- Instituto Español de Normalización.

13. Official Methods of Analysis of the AOAC.- 15th Edition (1990).- Method 963.22.

14. Ackman, R. G. and Sipos, J. C.- "Application of Specific Response Factors in the Gas Chromatographic Analysis of Methyl Esters of Fatty Acids with Flame Ionization Detectors".- J. Am. Oil Chemists' Soc. 41 (1964) 377-378.

(Recibido: Enero 1991) 